This item was submitted to Loughborough's Research Repository by the author.

Items in Figshare are protected by copyright, with all rights reserved, unless otherwise indicated.

\title{
Internal waves in a three-layer bubbly waveguide
}

PLEASE CITE THE PUBLISHED VERSION

LICENCE

CC BY-NC-ND 4.0

\section{REPOSITORY RECORD}

Grimshaw, Roger H.J., and Karima R. Khusnutdinova. 2019. "Internal Waves in a Three-layer Bubbly Waveguide". figshare. https://hdl.handle.net/2134/240. 


\title{
Internal waves in a three-layer bubbly waveguide
}

\author{
R.H.J. Grimshaw, K.R. Khusnutdinova * \\ Department of Mathematical Sciences, \\ Loughborough University, Loughborough LE11 3TU, UK \\ e-mails: \\ R.H.J.Grimshaw@lboro.ac.uk \\ K.Khusnutdinova@lboro.ac.uk
}

\begin{abstract}
In many oceanic situations, clouds, or layers of bubbles are present which may influence the larger-scale dynamics. A two-dimensional model of a diluted locally monodisperse mixture of an incompressible fluid with gas bubbles is used to model and study such situations. The problem is simplified by ignoring the horizontal variability in the structure of the bubble layer and by focusing instead on the vertical variability. We consider waves propagating horizontally in the oceanic waveguide, assuming that bubbles are confined to a thin upper layer of the otherwise homogeneous upper ocean, and using a three layer model to represent this situation. The presence of the depth-dependent distribution of bubbles introduces an effective stratification and considerably changes the value of the buoyancy frequency $N_{l}$ in the absence of bubbles, replacing it with an effective value $N$, where $N^{2}=N_{l}^{2}+g \alpha_{g 0}\left[\left(\ln n_{0}\right)_{z}+3 \alpha_{g 0}\left(\ln R_{0}\right)_{z}\right] /\left(1-\alpha_{g 0}\right)$ (here $\alpha_{g 0}$ is the void fraction, $n_{0}$ is the number density, and $R_{0}$ is the radius of bubbles in the basic state). This leads to the possibility of existence of the internal waves in the otherwise homogeneous upper mixed layer. Also the presence of the bubbly layer causes significant changes to the dispersion relation for the usual internal waves.
\end{abstract}

PACS numbers: 47.55.Hd, 47.55.Kf

Keywords: Internal waves, oceanic waveguide, bubbles

${ }^{*}$ On the leave from: Institute of Mechanics, Ufa Branch of the Russian Academy of Sciences, Karl Marx Str. 6, Ufa, 450000, Russia. 


\section{Introduction}

There is considerable observational and experimental evidence about the existence of bubble clouds, and, at higher wind speeds, of bubble layers in the upper ocean. The bubble distributions in the ocean surface layer have been studied in many different contexts: in connection with their role in air-sea gas transfer and as a measure of turbulence (Kolovaev 1976, Medwin 1977, Johnson and Cooke 1979, Thorpe 1982, Thorpe and Hall 1983, Thorpe 1984a, Woolf and Thorpe 1991, Thorpe 1995, Melville 1996, Farmer et al. 1999, Terrill and Melville 1999, Dean and Stokes 1999, Garrett et al. 2000), and also in connection with their influence on ambient noise (Medwin 1974, Urick 1983, Farmer and Lemon 1984, Farmer et al. 1997, Farmer and Vagle 1989, Buckingham 1997). Zedel and Farmer (1991) have observed the evolution of bubble layers during a storm.

The observed bubble distributions vary both as a function of time and depth and depend significantly on the wind speed. According to Thorpe (1982), at winds exceeding $6.5 \mathrm{~ms}^{-1}$ clouds of bubbles tend to overlap forming a continuous layer of variable thickness. According to Farmer and Vagle (1989), Zedel and Farmer (1991), Farmer et al. (1999), this highly structured bubble layer extends to a depth of several meters beneath the surface. Bubble concentration profiles have been measured in wind speeds of $11-13 \mathrm{~ms}^{-1}$ by Kolovaev (1976), Johnson and Cooke (1979), and Crawford and Farmer (1987). In general, there is a monotonic decrease in void fraction with increasing depth: the void fraction profile either decays exponentially or follows the inverse-square profile (Buckingham 1997). According to Farmer and Lemon (1984), the bubble concentration decreases exponentially with depth, with an $e$-folding scale of order $1 m$ which depends on the wind speed. A 20fold increase in bubble concentration was reported by Johnson and Cooke (1979) between the wind speed range $8.6-10.7$ and $11.8-13.9 \mathrm{~ms}^{-1}$. At wind speeds between 10 to $14 \mathrm{~ms}^{-1}$ the void fraction at the surface, averaged over a considerable period of time, is of order $10^{-7}-10^{-6}$ (see, e.g., Buckingham 1997 and the references therein, Farmer et al. 1999, Garrett et al. 2000). It can be larger than that for higher winds, or briefly so after the breaking of a wave. For example, at a wind speed of $15 \mathrm{~ms}^{-1}$ in observations by Terrill and Melville (1999), the void fractions approached levels of $10^{-4}$ at $0.69 \mathrm{~m}$ depth, while the highest densities measured at $4.1 \mathrm{~m}$ were approximately $5 \cdot 10^{-6}$. The mean void fraction at a depth of $0.69 \mathrm{~m}$ calculated for a 20 minute record was of order $10^{-5}$, while at $4.1 \mathrm{~m}$ it was of order $10^{-6}$. The distributions peak at all depths, and they decrease rapidly on either side of the peak and also rapidly with depth (see, e.g., Thorpe 1984b, Farmer et al. 1999 and the references there). The observations have shown that bubbles with radii of approximately $100 \mu \mathrm{m}$ contribute most to the total void fraction. In the observations reported by Farmer et al. (1999), in winds of approximately $12 \mathrm{~ms}^{-1}$ the peak radius declined from $130 \mu \mathrm{m}$ at $1.3 \mathrm{~m}$ to $70 \mu \mathrm{m}$ at $5.5 \mathrm{~m}$. In the observations made by Johnson and Cooke (1979) in winds of $11-13 \mathrm{~ms}^{-1}$ the peak was at the radius of about $50 \mu \mathrm{m}$ at all depths. According to Scott (1975), Thorpe (1984b) and Bullock et al. (2001) changes in salinity may have a profound effect on the bubble size distributions: far more small bubbles are formed in saline water than in fresh water.

As in our previous paper (Grimshaw and Khusnutdinova 2004), we follow an approach developed in the mechanics of multiphase media (see Nigmatulin 1991, Nakoryakov et al. 
1983, Kutateladze and Nakoryakov 1984, Prosperetti 1994, Brennen 1995 and references therein) to study the properties of the linear internal waves in a continuously and uniformly stratified fluid in the presence of bubbles. In the ocean surface layer bubbles occur mainly due to the breaking of surface waves, and evolve under the influence of buoyancy, turbulence, currents, dissolution and bubble break-up. Here, we consider the later stages of the fully-developed sea, when conditions can be assumed to be quasi-steady. Following Thorpe (1982), we assume that "the tendency of bubbles to rise is balanced by turbulent diffusion" and take the view that there is a steady-state bubble distribution in place, which is then perturbed. The processes of bubble formation, destruction and dissolution are not taken into account. Shear flow can be taken into account in our model (by choosing a more complicated basic state). However, here we follow the traditional approach towards studying internal waves and start from a simpler model, with no shear flow. Our present paper is organized as follows. In section 2 we formulate the mathematical model describing the two-dimensional motion of a diluted locally monodisperse mixture of incompressible fluid containing small gas bubbles. In Section 3 we briefly discuss the dispersion relation for the plane linear waves. In Section 4 we present some results for waves which are constrained to propagate horizontally in an oceanic waveguide, the upper part of which contains the depth-dependent distribution of bubbles. In our previous paper (Grimshaw and Khusnutdinova 2004) we used a two-layer model to represent this situation. Here, we make a more realistic assumption that bubbles are confined to a thin upper layer of the homogeneous upper ocean, and use a three layer model to obtain the dispersion relation, accounting for the mismatch between the depth of the bubble layer and the depth of the upper mixed layer. This also allows us to define the region of validity of our previously obtained results more precisely.

It should be mentioned, that, in recent years, bubbles have been intensively studied in many different situations. For instance, surface waves in a bubbly fluid, and waves on the interface between bubbly and ideal fluids in the absence of stratification were discussed by Akhatov (1989). Acoustic generation in a bubble layer has been studied in Druzhinin et al. (1996), Ostrovsky et al. (1998), Karpov et al. (2003). There also has been significant interest in studying the dynamics of clouds of bubbles in connection with cavitation problems (d'Agostino and Brennen 1989, Kumar and Brennen 1993, Brennen 1998, Reisman et al. 1998, see also references in Reisman et al. 1998). The relationship between internal waves and bubbles has been observed by Thorpe et al. (1987).

\section{Mathematical model}

We use a model describing the two-dimensional motion of a diluted locally monodisperse mixture of incompressible fluid containing small gas bubbles, preserving their mass and spherical form. Although the real oceanic flow is polydisperse, the use of a locally monodisperse model as an approximation to the real oceanic situation is based on the existence of a peak in the bubble distributions at all depths, as discussed in the Introduction. The distances between the bubbles are supposed to be big enough to prevent collisions, and so the interaction between bubbles is due only to pressure changes. The 
processes of bubble formation and destruction are not taken into account. The fluid phase is assumed to be incompressible, following the traditional approach towards studying internal waves (in the absence of bubbles). This is also justified by the great compressibility of bubbles compared to that of the surrounding liquid, and their small dimensions. The void fraction $\alpha_{g}$ is supposed to be small enough, $\alpha_{g} \ll 1$, so that the mass of the gas is neglected compared to the mass of the fluid. Thus, the mixture can be considered as a medium with the density approximately equal to $\rho_{l}\left(1-\alpha_{g}\right)$. Here, dissipative mechanisms are not taken into account (for a model accounting for the dissipation see Grimshaw and Khusnutdinova 2004).

Under these assumptions the set of equations describing a two-dimensional flow takes the form

$$
\begin{aligned}
& \rho \frac{d u}{d t}+p_{x}=0, \quad \rho \frac{d w}{d t}+p_{z}+\rho g=0, \\
& \frac{d \rho}{d t}+\rho\left(u_{x}+w_{z}\right)=0, \quad \frac{d n}{d t}+n\left(u_{x}+w_{z}\right)=0, \\
& \rho_{l}\left(R \frac{d^{2} R}{d t^{2}}+\frac{3}{2}\left(\frac{d R}{d t}\right)^{2}\right)=p_{g}-p-2 \frac{\sum}{R}, \\
& \frac{d \rho_{l}}{d t}=0, \rho=\rho_{l}\left(1-\alpha_{g}\right), \alpha_{g}=\frac{4}{3} \pi n R^{3}, \\
& \frac{d}{d t}\left(p_{g} R^{3 \kappa}\right)=0 .
\end{aligned}
$$

Here $p, \rho, u, w$ are pressure, density and velocity components of the mixture; $\rho_{l}$ is the density of a pure fluid; $p_{g}, \alpha_{g}, R, n$ are pressure, void fraction, radius and number density of bubbles; $\kappa$ is a polytropic exponent of a gas $(1 \leq \kappa \leq \gamma$, where $\gamma$ is the adiabatic exponent); $d / d t=\partial / \partial t+u \partial / \partial x+w \partial / \partial z$ is the material derivative with respect to time; and $\sum$ is the coefficient of surface tension. Here, the basic hydrodynamic equations for the mixture are closed by the Rayleigh-Plesset equation (3) for the oscillations of a single bubble, where the pressure at infinity is replaced by the pressure of the mixture (see Rayleigh 1917, Iordansky 1960, Kogarko 1961, Wijngaarden 1968, Plesset and Prosperetti 1977, Nigmatulin 1991). Equation (5) follows from the conservation laws for entropy of the gas, and the mass of the bubble, that is,

$$
\frac{d}{d t}\left(\ln p_{g} \rho_{g}^{-\kappa}\right)=0 \quad \text { and } \quad \frac{d}{d t}\left(\rho_{g} R^{3}\right)=0,
$$

respectively, where $\rho_{g}$ is the density of the gas. The details of derivation of equation (3), as well as discussion of the above model as a whole can be found, for example, in Nigmatulin (1991).

It is convenient to rewrite these equations in a different form, using (4). Indeed, from (4) we find that

$$
\frac{d \rho}{d t}=-\rho_{l} \frac{d \alpha_{g}}{d t}=-\frac{\rho}{1-\alpha_{g}} \frac{d \alpha_{g}}{d t} .
$$

From this, using the last relation in (4), we find that

$$
\frac{1}{\rho} \frac{d \rho}{d t}=-\frac{1}{1-\alpha_{g}}\left(\alpha_{g} \frac{1}{n} \frac{d n}{d t}+3 \alpha_{g} \frac{1}{R} \frac{d R}{d t}\right)
$$


while, from (2),

$$
\frac{1}{n} \frac{d n}{d t}=\frac{1}{\rho} \frac{d \rho}{d t}
$$

Finally, substituting (7) into (6), we obtain

$$
\frac{1}{\rho} \frac{d \rho}{d t}=-3 \alpha_{g} \frac{1}{R} \frac{d R}{d t} .
$$

Then, using (8), we can rewrite the governing equations (1) - (5) as follows:

$$
\begin{aligned}
& \rho \frac{d u}{d t}+p_{x}=0, \quad \rho \frac{d w}{d t}+p_{z}+\rho g=0, \\
& \frac{1}{\rho} \frac{d \rho}{d t}=\frac{1}{n} \frac{d n}{d t}=-3 \alpha_{g} \frac{1}{R} \frac{d R}{d t}, \\
& u_{x}+w_{z}=3 \alpha_{g} \frac{1}{R} \frac{d R}{d t}, \\
& \rho\left(R \frac{d^{2} R}{d t^{2}}+\frac{3}{2}\left(\frac{d R}{d t}\right)^{2}\right)=\left(1-\alpha_{g}\right)\left(p_{g}-p-2 \frac{\sum}{R}\right), \\
& \frac{d}{d t}\left(p_{g} R^{3 \kappa}\right)=0 .
\end{aligned}
$$

When written in this form, the governing equations can be more conveniently viewed as an extension, due to the bubbles, of the usual equations for the internal waves in incompressible fluid, which simplifies their analysis. Thus, the model we are working with consists of 7 equations (9) - (13) in 7 unknowns $p, \rho, u, w, R, n, p_{g}$, with $\alpha_{g}=4 \pi n R^{3} / 3$. In section 4 we will supplement these equations by boundary conditions at the ocean bottom and at the free surface in order to discuss horizontally propagating waves.

We will suppose that in the basic state the mixture has density $\rho_{0}(z)$, a corresponding pressure $p_{0}(z)$ (satisfying $p_{0 z}=-g \rho_{0}$ ), and that there is no shear flow, $u_{0}=w_{0}=0$. We also have $p_{g 0}(z)=p_{0}(z)+2 \sum / R_{0}$ and $\alpha_{g 0}=4 \pi n_{0} R_{0}^{3} / 3$. Here, we take into consideration only the vertical variability of the bubble layer and do not consider the horizontal spatial variations of its thickness, which in reality is a significant feature of the upper ocean. However, we think that this is a correct idealization of the real situation, allowing us to obtain a simplified problem and to focus on the effects due to the vertical variability of the bubble layer.

Using the Bousinesq approximation, the linearized equations describing deviations from the basic state take the form

$$
\begin{aligned}
& \rho_{0} \tilde{u}_{t}+\tilde{p}_{x}=0 \\
& \rho_{0} \tilde{w}_{t}+\tilde{p}_{z}+g \tilde{\rho}=0, \\
& \tilde{\rho}_{t}+\rho_{0 z} \tilde{w}=-3 \frac{\alpha_{g 0} \rho_{0}}{R_{0}}\left(\tilde{R}_{t}+R_{0 z} \tilde{w}\right), \\
& \tilde{u}_{x}+\tilde{w}_{z}=3 \frac{\alpha_{g 0}}{R_{0}}\left(\tilde{R}_{t}+R_{0 z} \tilde{w}\right), \\
& \rho_{0} R_{0}\left(\tilde{R}_{t t}+R_{0 z} \tilde{w}_{t}\right)=\left(1-\alpha_{g 0}\right)\left(\tilde{p}_{g}-\tilde{p}+2 \frac{\sum}{R_{0}^{2}} \tilde{R}\right),
\end{aligned}
$$




$$
\begin{aligned}
& \tilde{p}_{g t}+\frac{3 \kappa p_{g 0}}{R_{0}} \tilde{R}_{t}+\frac{\left(p_{g 0} R_{0}^{3 \kappa}\right)_{z}}{R_{0}^{3 \kappa}} \tilde{w}=0, \\
& \tilde{n}_{t}+n_{0 z} \tilde{w}=-3 \frac{\alpha_{g 0} n_{0}}{R_{0}}\left(\tilde{R}_{t}+R_{0 z} \tilde{w}\right) .
\end{aligned}
$$

In the sequel, the tilde superscripts are omitted. Note that $n$ appears only in the last equation, and so doesn't influence the dispersion relation.

It is convenient to work with the equations involving only the vertical velocity $w$, and $s=R_{t}$, the radial velocity of the bubbles (which in this model is assumed to be described by one function for all bubbles). The derivation of these equations is given below. The horizontal velocity $u$ can be eliminated by taking the time derivative of (17) and using the horizontal momentum equation (14), which leads to

$$
\frac{1}{\rho_{0}} p_{x x}=w_{z t}-\frac{3 \alpha_{g 0}}{R_{0}}\left(R_{t t}+R_{0 z} w_{t}\right) .
$$

Then on taking the time derivative of the vertical momentum equation (15) and eliminating $\rho$ by virtue of (16), we get

$$
\begin{aligned}
& \frac{1}{\rho_{0}} p_{z t}=-w_{t t}-N^{2} w+\frac{3 \alpha_{g 0} g}{R_{0}} R_{t}, \\
& \text { where } \quad N^{2}=-g\left(\frac{1}{\rho_{0}} \frac{d \rho_{0}}{d z}+\frac{3 \alpha_{g 0}}{R_{0}} \frac{d R_{0}}{d z}\right) .
\end{aligned}
$$

The pressure $p$ can be eliminated by taking the second derivative of (22) with respect to $x$, and using (21). In the Boussinesq approximation we obtain

$$
\frac{\partial^{2}}{\partial t^{2}} \triangle w+N^{2} w_{x x}-\left[b\left(s_{t t}+R_{0 z} w_{t t}\right)\right]_{z}-g b s_{x x}=0,
$$

where $\triangle \equiv \partial^{2} / \partial x^{2}+\partial^{2} / \partial z^{2}$ is the two-dimensional Laplacian, and $b=3 \alpha_{g 0} / R_{0}$. Here the effective buoyancy frequency $N$ (see 23 ) is given by

$$
N^{2}=N_{l}^{2}+g\left(\frac{\alpha_{g 0_{z}}}{1-\alpha_{g 0}}-3 \alpha_{g 0} \frac{R_{0 z}}{R_{0}}\right)=N_{l}^{2}+\frac{g \alpha_{g 0}}{1-\alpha_{g 0}}\left[\left(\ln n_{0}\right)_{z}+3 \alpha_{g 0}\left(\ln R_{0}\right)_{z}\right],
$$

where $N_{l}$ is the usual buoyancy frequency of the liquid (in the absence of bubbles). Here, we have used the relation $\alpha_{g 0}=4 \pi n_{0} R_{0}^{3} / 3$. When the void fraction $\alpha_{g 0} \ll 1$, the term involving the number density gradient $\left(\ln n_{0}\right)_{z}$ dominates that due to the gradient of the bubble radius $\left(\ln R_{0}\right)_{z}$. Since $N_{l}^{2}$ in the upper mixed layer is usually very small and $\left(\ln n_{0}\right)_{z}$ is of order 1 , then even for $\alpha_{g 0}$ of order $10^{-8}$, the effective buoyancy frequency $N$ significantly differs from the value of the buoyancy frequency $N_{l}$ in the absence of any bubbles. For larger void fractions of bubbles (for example, for $\alpha_{g 0} \sim 10^{-6}-10^{-5}$, which is not a rare occurrence in the ocean) we can get anomalous high values of the effective buoyancy frequency in the upper ocean, which leads to the possibility of existence of subsurface internal waves similar to the usual internal waves in deep water (see also the end of Section 4). Note that this significant influence of bubbles on internal waves manifests itself already in the linear approximation. 
A second equation can be derived by differentiating the linearized Rayleigh-Plesset equation (18) twice by $x$ and once by $t$, and then using (19) and (21). This leads to

$$
\begin{aligned}
& s_{t t x x}+\omega_{*}^{2}\left(1-\alpha_{g 0}\right) s_{x x}-a s_{t t}+ \\
& \frac{a}{b}\left(w_{t t z}+\frac{\left(p_{g 0} R_{0}^{3 \kappa}\right) z}{\rho_{0} R_{0}^{3 \kappa}} w_{x x}\right)+R_{0 z}\left(w_{x x}-a w\right)_{t t}=0, \\
& \text { where } \quad \omega_{*}^{2}=\frac{3 \kappa p_{0}}{R_{0}^{2} \rho_{0}}+\frac{2 \sigma(3 \kappa-1)}{R_{0}^{3}}
\end{aligned}
$$

is the natural frequency of bubble oscillations, $\sigma=\sum / \rho_{0}$, and $a=3 \alpha_{g 0}\left(1-\alpha_{g 0}\right) / R_{0}^{2}$. Note that if $\sigma=0$, then $\omega_{*}^{2}=3 \kappa p_{0} / R_{0}^{2} \rho_{0}$ is a Minnaert frequency (Minnaert 1933).

We now assume that the radius $R_{0}$ of bubbles in the basic state is independent of the depth. This approximation is based on the observations discussed in the Introduction, from which we argue that the changes in the number of bubbles with depth are much more crucial. Then, introducing the new variable (the depth-dependent scaling of the radial velocity of the bubble),

$$
c=\alpha_{g 0}(z) s
$$

equations (24) and (25) can be brought to the form

$$
\begin{aligned}
& \frac{\partial^{2}}{\partial t^{2}} \Delta w+N^{2} w_{x x}-\delta\left(c_{t t z}+g c_{x x}\right)=0, \\
& c_{t t x x}+\omega_{*}^{2}\left(1-\alpha_{g 0}\right) c_{x x}-a c_{t t}+\frac{a}{\delta}\left(w_{t t z}-g w_{x x}\right)=0 .
\end{aligned}
$$

Here $\delta=3 / R_{0}$. We shall next simplify the analysis by assuming that the effective buoyancy frequency $N(23)$ is constant, at least in each of several layers (see Section 4). This then gives us $\rho_{0}=\rho_{a} \exp \left(-N^{2} z / g\right)$ and $p_{0}=p_{a}+g^{2}\left(\rho_{0}-\rho_{a}\right) / N^{2}$, where $p_{a}$ and $\rho_{a}$ are constants, which may be taken as the atmospheric pressure and the density at the free surface respectively. However, even in the case of constant $N, \omega_{*}(26)$ and the parameter $\alpha_{g 0}$ in the coefficients of (27) depend on $z$. In our present analysis we consider a simplified model by supposing that the values of $\omega_{*}$ and $\alpha_{g 0}$ in the coefficients of (27) are constant and equal to their average value over the depth of the bubble layer. Thus, in this simplified model, the depth-dependence of the actual distribution of bubbles is taken into account only by introducing the effective buoyancy frequency $N$. We shall also make the usual assumption that $\sigma$ is a constant.

\section{Dispersion relation}

The first step is to obtain the dispersion relation, by seeking solutions in the form of propagating waves. Considering two-dimensional plane waves

$$
\left(\begin{array}{l}
w \\
c
\end{array}\right)=\left(\begin{array}{l}
W \\
C
\end{array}\right) e^{i(k x+l z-\omega t)}+\text { c.c. }
$$


we obtain the dispersion relation

$$
a_{1} \omega^{4}-a_{2} \omega^{2}+a_{3}=0
$$

where the coefficients are given by

$$
\begin{aligned}
& a_{1}=k^{2}+l^{2}+a, \\
& a_{2}=N^{2}\left(k^{2}+a\right)+\omega_{*}^{2}\left(1-\alpha_{g 0}\right)\left(k^{2}+l^{2}\right), \\
& a_{3}=\left(\omega_{*}^{2} N^{2}\left(1-\alpha_{g 0}\right)-a g^{2}\right) k^{2} .
\end{aligned}
$$

From (29) we obtain

$$
\omega_{ \pm}^{2}=\frac{a_{2} \pm \sqrt{D}}{2 a_{1}}, D=a_{2}^{2}-4 a_{1} a_{3} .
$$

The expression for the discriminant $D$ can be rewritten as

$$
D=\left(\omega_{*}^{2}\left(1-\alpha_{g 0}\right)\left(k^{2}+l^{2}\right)-N^{2}\left(k^{2}+a\right)\right)^{2}+4 a g^{2} k^{2}\left(k^{2}+l^{2}+a\right)+4 \omega_{*}^{2}\left(1-\alpha_{g 0}\right) N^{2} a l^{2},
$$

showing that $D>0$. Next, we observe that if $a_{3}<0$, then $\omega_{-}^{2}<0$ implying the existence of a temporal instability. However, since $a_{3}<0$ is equivalent to $\omega_{*}^{2} R_{0}^{2} N^{2}<3 \alpha_{g 0} g^{2}$, it is unlikely to be satisfied under the hypotheses we have used in deriving this set of equations. Therefore, we shall always assume that $a_{3}>0$. In this case, $D<a_{2}^{2}$, and (30) shows that the dispersion relation has two branches, $\omega_{+}$and $\omega_{-}$, characterized by the following properties.

Let the vertical wavenumber $l$ be fixed. Then, as $k \rightarrow \infty$,

$$
\omega_{ \pm}^{2} \rightarrow \omega_{ \pm(\infty)}^{2}=\frac{1}{2}\left(\omega_{*}^{2}\left(1-\alpha_{g 0}\right)+N^{2} \pm \sqrt{\left(\omega_{*}^{2}\left(1-\alpha_{g 0}\right)-N^{2}\right)^{2}+4 a g^{2}}\right) .
$$

Taking into account that for typical values of parameters $4 a g^{2} \ll\left(\omega_{*}^{2}\left(1-\alpha_{g 0}\right)-N^{2}\right)^{2}$ and $N^{2} \ll \omega_{*}^{2}\left(1-\alpha_{g 0}\right)$, we get

$$
\begin{aligned}
& \omega_{+(\infty)}^{2} \approx \omega_{*}^{2}\left(1-\alpha_{g 0}\right)+\frac{3 \alpha_{g 0} g^{2}}{\omega_{*}^{2} R_{0}^{2}}, \\
& \omega_{-(\infty)}^{2} \approx N^{2}-\frac{3 \alpha_{g 0} g^{2}}{\omega_{*}^{2} R_{0}^{2}} .
\end{aligned}
$$

On the other hand, as $k \rightarrow 0$,

$$
\begin{aligned}
& \omega_{+}^{2} \rightarrow \omega_{+(0)}^{2}=\frac{\omega_{*}^{2}\left(1-\alpha_{g 0}\right) l^{2}+a N^{2}}{l^{2}+a}, \\
& \omega_{-}^{2} \rightarrow \omega_{-(0)}^{2}=0 .
\end{aligned}
$$

When $k$ is fixed and $l \rightarrow \infty$, then

$$
\omega_{+}^{2} \rightarrow \omega_{*}^{2}\left(1-\alpha_{g 0}\right), \quad \omega_{-}^{2} \rightarrow 0 .
$$


If $\alpha_{g 0} \rightarrow 0$, then

$$
\omega_{+}^{2}=\omega_{*}^{2} \quad \text { and } \quad \omega_{-}^{2}=\frac{N^{2} k^{2}}{k^{2}+l^{2}} .
$$

Thus, $\omega_{+}$corresponds to the natural frequency of bubble oscillations, while $\omega_{-}$gives us the dispersion relation for the internal waves in a pure stratified fluid. When $\alpha_{g 0}>0$, corresponding to the presence of bubbles, we see that the frequency of an internal wave lies in the band $0 \leq \omega<\omega_{-(\infty)}$, and, for a fixed wavenumber $(k, l)$, significantly differs from the value in the absence of bubbles (for the uniform distribution of bubbles it is reduced). For the depth-dependent distribution of bubbles, the frequency of an internal waves is reduced from the value of the effective buoyancy frequency (23). For example, considering the typical oceanic situation, let the buoyancy frequency of the liquid be $N_{l}=10^{-3} \mathrm{~s}^{-1}$ and the mean void fraction of bubbles be $\alpha_{g 0}=5 \cdot 10^{-6}$. Supposing that $\left(\ln n_{0}\right)_{z} \approx 1$, we estimate the value of the effective buoyancy frequency as $N \approx 7.07 \cdot 10^{-3} s^{-1}$. For $\omega_{*}=2.26 \cdot 10^{5} \mathrm{~s}^{-1}$ and $R_{0}=100 \mu \mathrm{m}$, the frequency of an internal wave is bounded by a slightly reduced value of $\omega_{-(\infty)} \approx 6.87 \cdot 10^{-3} s^{-1}$. This value significantly differs from the value $N_{l}=10^{-3} s^{-1}$ in the absence of bubbles. The "bubble" wave frequency lies in the band $\omega_{+(0)} \leq \omega<\omega_{+(\infty)}$. Since $\omega_{+(0)} \geq N>\omega_{-(\infty)}$, there is a gap in the frequency spectrum, and this gap contains the effective buoyancy frequency $N$ (23). In particular, the limiting frequency for an internal wave is $\omega_{-(\infty)}$ which is less than $N$.

The group velocity for each branch is $(\partial \omega / \partial k, \partial \omega / \partial l)$, and can be written in the form,

$$
\begin{aligned}
\frac{\partial \omega_{ \pm}}{\partial k} & =\frac{k}{\omega_{ \pm}} \cdot \frac{\left(\omega_{ \pm}^{2}-\omega_{+(\infty)}^{2}\right)\left(\omega_{ \pm}^{2}-\omega_{-(\infty)}^{2}\right)}{\mp \sqrt{D}}, \\
\frac{\partial \omega_{ \pm}}{\partial l} & =\omega_{ \pm} l \cdot \frac{\omega_{ \pm}^{2}-\omega_{*}^{2}\left(1-\alpha_{g 0}\right)}{\mp \sqrt{D}}
\end{aligned}
$$

It follows that (assuming without loss of generality that $\omega_{ \pm}>0$ ) for the internal wave, the horizontal component of group velocity is always positive, and the vertical component is always negative (as for the case when there are no bubbles). For the bubble mode, both the horizontal and the vertical components of group velocity are positive, except for short waves $(k \rightarrow \infty)$ for which $\omega_{+}^{2}>\omega_{*}^{2}\left(1-\alpha_{g 0}\right)$ when the vertical component is negative. Further, it can be shown that for the internal wave, the horizontal component of group velocity is reduced from that in a pure fluid with the buoyancy frequency equal to the effective value (23), while the absolute value of the vertical component is increased, except for very long waves for which $k, l \rightarrow 0$.

If $\alpha_{g 0} \rightarrow 0$, then (31) yields

$$
\left(\frac{\partial \omega_{-}}{\partial k}, \frac{\partial \omega_{-}}{\partial l}\right) \rightarrow \frac{l \omega_{-}}{k\left(k^{2}+l^{2}\right)}(l,-k),
$$

which is the group velocity for internal waves in a pure stratified fluid, and is normal to the phase velocity. Let us note, that in the presence of bubbles the group velocity is no longer normal to the phase velocity, since, as can be seen from (17), the particle motion is not normal to the wavenumber vector. Further, for $l=0$, the group velocity of internal 
waves in a pure fluid is zero, but in the presence of bubbles it has a nonzero horizontal component.

Note, that if we put $g=0$ in our formulas, the dispersion relation collapses to $\omega^{2}=0$ (the remnant of the internal wave branch) and a single non-trivial branch,

$$
\omega^{2}=\frac{\omega_{*}^{2}\left(k^{2}+l^{2}\right)}{k^{2}+l^{2}+a} .
$$

For long waves (i.e. $\left.k^{2}+l^{2} \ll a\right)(32)$ yields the well-known formula for the sound velocity in a gas-liquid mixture (e.g., Naugolnykh and Ostrovsky 1998):

$$
\omega^{2} \approx c_{0}^{2}\left(k^{2}+l^{2}\right), \quad c_{0}^{2}=\frac{\kappa p_{0}}{\rho_{0} \alpha_{g 0}} .
$$

Thus, returning to the dispersion relation (30) we can say that the bubble mode (the upper branch of the dispersion relation) can be interpreted as modified acoustic waves, while the lower branch represents modified internal waves (but note that for very long waves there is an exchange of identity between the branches). Although the liquid is assumed to be incompressible, the presence of bubbles gives the mixture some compressibility.

\section{Waves in a three-layer bubbly waveguide}

We now consider the oceanic waveguide, and suppose that the fluid is bounded below by a flat rigid bottom, and above by a free surface. Here, we assume that there is a thin upper layer of the homogeneous upper ocean which has a bubble distribution, and there are no bubbles in the rest of the upper mixed layer and in the deep ocean. We use a three-layer model, shown in Fig. 1, to represent this situation.

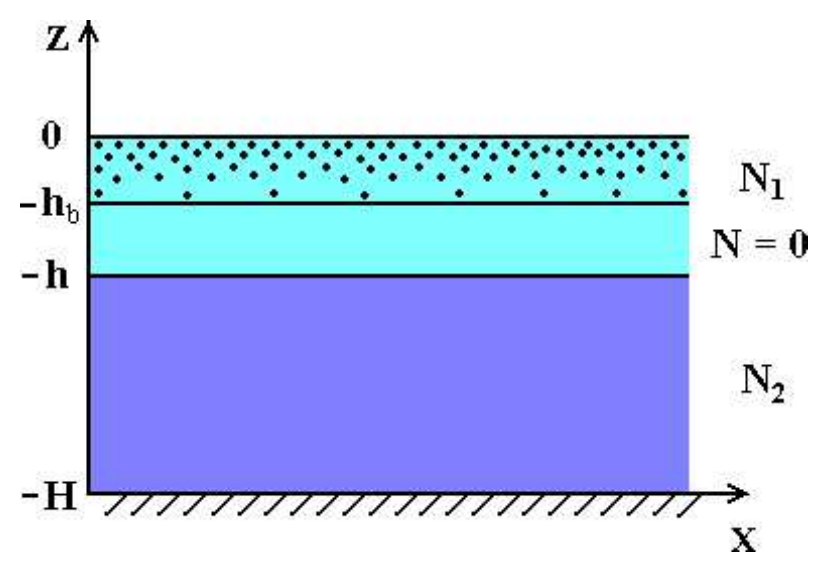

Figure 1: The three-layer bubbly waveguide.

The upper bubbly layer has an undisturbed constant depth $h_{b}$, a constant effective buoyancy frequency $N_{1}$ and contains bubbles. This bubble layer is a part of the otherwise 
homogeneous upper mixed layer, which has an undisturbed constant depth $h$. The lower layer is without bubbles, and has a constant buoyancy frequency $N_{2}$. It is important to note, that although we assume the upper layer to be homogeneous in the absence of bubbles, the effective buoyancy frequency $N_{1}$ can be comparable (or even bigger) than the buoyancy frequency $N_{2}$ in the lower stratified pure fluid layer. The density $\rho_{0}(z)$ is assumed to be continuous at both interfaces: $z=-h_{b}$ and $z=-h$. In the sequel the subscript 1 corresponds to the upper bubble layer, the subscript 2 to the middle homogeneous pure liquid layer, and the subscript 3 to the lower stratified pure liquid layer. The linearized equations of motion are again (14)-(20). The bottom boundary condition is

$$
\left.w_{3}\right|_{z=-H}=0,
$$

where $H$ is the undisturbed fluid depth. In the Boussinesq approximation for internal waves, we may replace the free-surface boundary conditions with the so-called "rigid-lid" condition (e.g., Gill 1982),

$$
\left.w_{1}\right|_{z=0}=0 .
$$

The linearized conditions at the first interface (between the upper bubble layer and the middle layer) are

$$
\begin{aligned}
& \left.w_{1}\right|_{z=-h_{b}}=\left.w_{2}\right|_{z=-h_{b}}=\eta_{t}, \\
& \left.p_{1}\right|_{z=-h_{b}}=\left.p_{2}\right|_{z=-h_{b}},
\end{aligned}
$$

expressing continuity of the vertical displacement and the pressure (in (36), we have taken into account that there is no density jump at the interface). Here, $\eta(x, t)$ is the first interface displacement. Similarly, the linearized conditions at the second interface (between the middle layer and the lower layer) are

$$
\begin{aligned}
& \left.w_{2}\right|_{z=-h}=\left.w_{3}\right|_{z=-h}=\zeta_{t}, \\
& \left.p_{2}\right|_{z=-h}=\left.p_{3}\right|_{z=-h},
\end{aligned}
$$

where $\zeta(x, t)$ is the second interface displacement.

In the lower stratified layer (pure fluid without bubbles) we have the equation

$$
\frac{\partial^{2}}{\partial t^{2}} \triangle w_{3}+N_{2}^{2} w_{3_{x x}}=0 .
$$

The solution of (39), which satisfies (33) has the form

$$
\begin{aligned}
& w_{3}=A \sin l(z+H) \exp [i(k x-\omega t)]+c . c ., \\
& \text { where } \quad \omega^{2}=\frac{N_{2}^{2} k^{2}}{k^{2}+l^{2}}
\end{aligned}
$$

is the dispersion relation in a pure stratified fluid. For an internal wave we assume that $l^{2}>0$ and so then $\omega^{2}<N_{2}^{2}$, although the following analysis remains valid if instead $l^{2}<0$. In the homogeneous middle layer we have

$$
w_{2}=(B \sinh k z+C \cosh k z) \exp [i(k x-\omega t)]+c . c .
$$


In the upper bubble layer we have equations (27) as in section 3, but with $N$ replaced by $N_{1}$. Here we assume that the coefficients $\omega_{*}, \alpha_{g 0}$ are replaced by constants, equal to their average value over the depth of the bubble layer. To the leading order in the Boussinesq approximation, we have

$$
\omega_{*}^{2} \approx \frac{3 \kappa}{R_{0}^{2}}\left(\frac{p_{a}}{\rho_{a}}+\frac{g h_{b}}{2}\right)+\frac{2 \sigma(3 \kappa-1)}{R_{0}^{3}} .
$$

Here $p_{a}$ is the atmospheric pressure, and $\rho_{a}$ is the density at the free surface. The solutions of (27) can be found in the form

$$
\begin{aligned}
& w_{1}=\phi(z) \exp [i(k x-\omega t)]+c . c . \\
& c=\psi(z) \exp [i(k x-\omega t)]+c . c .
\end{aligned}
$$

Substituting (43) into (27) we obtain

$$
\phi^{\prime \prime}-\beta^{2} \phi=0, \quad \psi=\alpha\left(\omega^{2} \phi^{\prime}-g k^{2} \phi\right),
$$

where

$$
\beta^{2}=\frac{\left(\omega^{2}-N_{1}^{2}-\alpha \delta g^{2} k^{2}\right) k^{2}}{\omega^{2}\left(1-\alpha \delta \omega^{2}\right)}, \quad \alpha=\frac{a}{\delta\left[\omega^{2}\left(k^{2}+a\right)-\omega_{*}^{2}\left(1-\alpha_{g 0}\right) k^{2}\right]} .
$$

It is convenient to assume that $\beta^{2}>0$ which is the natural choice for internal waves when $N_{1}<N_{2}$. However, in fact the following analysis remains valid if $\beta^{2}<0$, and situations when this occurs will be discussed below. Thus equation (44) has the solution

$$
\phi(z)=D \sinh \beta z,
$$

satisfying condition (34).

Next, to apply the boundary conditions at the interfaces we let $\eta(x, t)=i \tilde{a} \exp [i(k x-$ $\omega t)]+$ c.c. and $\zeta(x, t)=i \tilde{b} \exp [i(k x-\omega t)]+c . c$. Substituting (43) and (42) into (35), we obtain

$$
D=-\frac{\tilde{a} \omega}{\sinh \beta h_{b}}, \quad B \sinh k h_{b}-C \cosh k h_{b}=-\tilde{a} \omega .
$$

Condition (36) can be rewritten in terms of the variables $w_{1}, c, w_{2}$ by differentiating (36) twice with respect to $x$ and using (21) in the bubbly layer, and the similar condition with $\alpha_{g 0}=0$ in the pure fluid. We obtain

$$
w_{1_{z t}}-\left.\delta c_{t}\right|_{z=-h_{b}}=\left.w_{2_{z t}}\right|_{z=-h_{b}},
$$

which leads to

$$
\left(1-\alpha \delta \omega^{2}\right) \beta \operatorname{coth} \beta h_{b}-k \frac{B \cosh k h_{b}-C \sinh k h_{b}}{B \sinh k h_{b}-C \cosh k h_{b}}=\alpha \delta g k^{2} .
$$

Now, substituting (42) and (40) into (37), we obtain

$$
A=\frac{\tilde{b} \omega}{\sin l(H-h)}, \quad B \sinh k h-C \cosh k h=-\tilde{b} \omega .
$$


As before, we can rewrite (38) in terms of the variables $w_{2}$ and $w_{3}$ :

$$
\left.w_{2_{z t}}\right|_{z=-h}=\left.w_{3_{z t}}\right|_{z=-h},
$$

which gives us

$$
k(B \cosh k h-C \sinh k h)=A l \cos l(H-h) .
$$

Finally, defining $B$ and $C$ from (47) and (48), and substituting the values of $B$ and $C$ into (46), we obtain the dispersion relation,

$$
\begin{gathered}
\left(1-\alpha \delta \omega^{2}\right) \beta \operatorname{coth} \beta h_{b}-\alpha \delta g k^{2}= \\
-l \frac{\cos (H-h) l \cosh \left(h-h_{b}\right) k+\frac{k}{l} \sin (H-h) l \sinh \left(h-h_{b}\right) k}{\sin (H-h) l \cosh \left(h-h_{b}\right) k+\frac{l}{k} \cos (H-h) l \sinh \left(h-h_{b}\right) k},
\end{gathered}
$$

accounting for the mismatch between the depth of the bubble layer and the depth of the upper mixed layer. Note that $k^{2}=\omega^{2} l^{2} /\left(N_{2}^{2}-\omega^{2}\right)$, and, therefore, from (45), we obtain

$$
\begin{aligned}
& 1-\alpha \delta \omega^{2}=\frac{\left(\omega_{*}^{2}\left(1-\alpha_{g 0}\right)-\omega^{2}\right) l^{2}}{\left(\omega_{*}^{2}\left(1-\alpha_{g 0}\right)-\omega^{2}\right) l^{2}-a\left(N_{2}^{2}-\omega^{2}\right)}, \\
& \alpha \delta k^{2}=-\frac{a l^{2}}{\left(\omega_{*}^{2}\left(1-\alpha_{g 0}\right)-\omega^{2}\right) l^{2}-a\left(N_{2}^{2}-\omega^{2}\right)},
\end{aligned}
$$

and

$$
\beta^{2}=K_{1} l^{2}+K_{2}
$$

where $K_{1}$ and $K_{2}$ are given by

$$
\begin{aligned}
K_{1} & =\frac{\omega^{2}-N_{1}^{2}}{N_{2}^{2}-\omega^{2}}+\frac{a g^{2}}{\left(N_{2}^{2}-\omega^{2}\right)\left(\omega_{*}^{2}\left(1-\alpha_{g 0}\right)-\omega^{2}\right)}, \\
K_{2} & =\frac{a\left(N_{1}^{2}-\omega^{2}\right)}{\omega_{*}^{2}\left(1-\alpha_{g 0}\right)-\omega^{2}} .
\end{aligned}
$$

Thus, for a given frequency $\omega$, the dispersion relation (49) is an equation for $l$. In the limiting case of $h_{b} \rightarrow h$, (49) gives us the dispersion relation for a two-layer model, obtained earlier (Grimshaw and Khusnutdinova 2004). In the limiting case of $h_{b} \rightarrow 0$ (or $h_{b} \rightarrow h$ and $\alpha \delta \rightarrow 0$ ), (49) gives us the dispersion relation for a two-layer pure fluid (no bubbles).

Next, we assume that $|\beta| h_{b} \ll 1$ (the upper bubbly layer is thin) and

$$
\nu l \ll 1, \quad \text { where } \quad \nu=\frac{\omega\left(h-h_{b}\right)}{\sqrt{N_{2}^{2}-\omega^{2}}}
$$

(for $\omega<N_{2}$ this means that the vertical wavenumber $l$ is small compared to $\nu^{-1}$ ). Note that if $h-h_{b} \rightarrow 0$, then $\nu^{-1} \rightarrow \infty$. Then (49) can be approximated by

$$
b_{1}\left[\tan (H-h) l+\left(h-h_{b}\right) l\right]+h_{b}\left(b_{2} l-\frac{b_{3}}{l}\right)=0,
$$


where the coefficients are given by

$$
\begin{aligned}
& b_{1}=\omega_{*}^{2}\left(1-\alpha_{g 0}\right)-\omega^{2}+a g h_{b}, \\
& b_{2}=\omega_{*}^{2}\left(1-\alpha_{g 0}\right)-\omega^{2}, \quad b_{3}=a\left(N_{2}^{2}-\omega^{2}\right) .
\end{aligned}
$$

For a given frequency $\omega$ and the given system parameters, the values of $l$ determined by (51) are restricted to a discrete set of values (which depends on the frequency and system parameters).

For $h / H \ll 1$ we further simplify (51) and obtain the approximate solutions. Up to the first correction in $h_{b} / H$ these solutions are given by

$$
\begin{aligned}
& l_{n} \approx l_{n}^{0}+\frac{h_{b}}{H} \tilde{l}_{n}, \quad n=1,2, \ldots, \\
& \text { where } \quad \tilde{l}_{n}=\frac{a l_{n}^{0}}{b_{1}}\left(\frac{N_{2}^{2}-\omega^{2}}{l_{n}^{02}}+g h_{b}\right), \quad l_{n}^{0}=\frac{n \pi}{H},
\end{aligned}
$$

together with the additional value $l_{0}$ given by

$$
l_{0}^{2} \approx \frac{b_{3} h_{b}}{b_{1} H-a g h_{b}^{2}},
$$

which can be interpreted as a bubble mode, as it does not exist if the upper layer has no bubbles. The modes with $n \geq 1$ can be interpreted as internal wave modes, which all exist when there are no bubbles. Note that these approximate solutions depend on the depth of the bubble layer and do not depend on the depth of the upper mixed layer. Therefore, the results obtained in our previous paper (Grimshaw and Khusnutdinova 2004) remain valid. However, the region of the validity of these results is now defined more precisely, by imposing the additional condition (50). Note that the value of $l_{n}$, for a given frequency $\omega<N_{2}$ is increased due to the presence of the upper layer when bubbles are present (as then $\left.\tilde{l}_{n}>0\right)$. It is interesting to note that if there are no bubbles in the upper layer, then $\tilde{l}_{n}$ is zero, and the correction to the leading order term $n \pi / H$ for $l_{n}$ is then $O\left(h^{3} / H^{3}\right)$ ( $l_{n} \approx n \pi / H+(n \pi)^{3} h^{3} / 3 H^{4}$, so that the correction is still positive).

For each mode, the dispersion relation expressing $\omega$ as a function of $k$ is obtained by combining the expressions (41) and (52). It can then be solved for $\omega$ as a function of $k$. For the bubble mode $\left(l=l_{0}\right)$, this gives the approximate relation

$$
\omega^{2}=\frac{\left[\omega_{*}^{2}\left(1-\alpha_{g 0}\right) H+a g h_{b}\left(H-h_{b}\right)\right] k^{2}}{a h_{b}+H k^{2}} .
$$

Note that, as $k^{2}$ increases, so does $\omega^{2}$, and hence there is a critical value $k_{N}$ (obtained by setting $\omega^{2}=N_{2}^{2}$ in (53)), such that for $k>k_{N}, l_{0}^{2}<0$ and the mode is evanescent in the lower layer, but for $k<k_{N}, l_{0}^{2}>0$ and the mode is then sinusoidal in the lower layer. In practice $k_{N} H \ll 1$ and so this latter possibility is confined to very long waves.

For internal waves in the lower layer, the dispersion relation is just (41) with $l \approx n \pi / H$ to leading order in $h_{b} / H$. Thus for these modes, $l^{2}>0$ and so $\omega^{2}<N_{2}^{2}$. The small correction given by $\tilde{l}_{n}(52)$ has the effect of slightly increasing the wavenumber $k$, for a 
given $\omega$, due to the presence of the bubbles in the upper layer, or equivalently of decreasing the frequency $\omega$ for a given wavenumber $k$. Further, this effect is $O\left(h_{b} / H\right)$ and for high void fractions and small ratios $h / H$ it may be comparable with the corresponding effect in the absence of any bubbles, when it is $O\left(h^{3} / H^{3}\right)$. For example, if $h_{b}=10 \mathrm{~m}, h=$ $50 \mathrm{~m}, H=4000 \mathrm{~m}, R_{0}=100 \mu \mathrm{m}, \omega_{*}=2.5 \cdot 10^{5} \mathrm{~s}^{-1}, N_{2}=10^{-3} \mathrm{~s}^{-1}, \omega=0.5 N_{2}$, we obtain $\left(l_{1}-l_{1}^{0}\right) / l_{1}^{0} \approx 1.2 \cdot 10^{-3} \alpha_{g 0}$, and this can be comparable with the corresponding correction in the absence of any bubbles, when it is $O\left(10^{-6}\right)$ (the corresponding value in the framework of the two-layer model with $h=h_{b}=10 \mathrm{~m}$ was $\left.O\left(10^{-8}\right)\right)$. On the other hand, in shallow water, for $H=100 \mathrm{~m}$ and $h=h_{b}=10 \mathrm{~m}$ say, $\left(l_{1}-l_{1}^{0}\right) / l_{1}^{0} \approx 5 \cdot 10^{-2} \alpha_{g 0}$. This is smaller than the corresponding correction in the absence of any bubbles, which is $O\left(10^{-3}\right)$, but can also be significant if the void fraction is high enough.

Next we consider the case when $N_{2}$ is zero, so that in effect the stratification is confined to the upper bubble layer, and is due to the depth-dependent distribution of bubbles only. We then have $l=i k$. We also now relax the hypothesis that $|\beta| h_{b} \ll 1$. To allow for the waves to be sinusoidal in the upper bubble layer we now set $\beta=i \tilde{\beta}$. The dispersion relation (49) then takes the form,

$$
\left(1-\alpha \delta \omega^{2}\right) \tilde{\beta} \cot \tilde{\beta} h_{b}+k \operatorname{coth}\left(H-h_{b}\right) k=\alpha \delta g k^{2},
$$

where, from (45),

$$
k^{2}=K_{1}^{-1} \tilde{\beta}^{2}+\frac{K_{2}}{K_{1}} .
$$

Therefore, the dispersion relation (54) has a structure

$$
\tilde{\beta} \cot \tilde{\beta} h_{b}=F(\tilde{\beta}, \omega),
$$

where, for each fixed $\omega$ and for all $k^{2}>0, F$ is a continuous function of $\tilde{\beta}^{2}$. Since $\cot \tilde{\beta} h_{b}$ will pass from $+\infty$ to $-\infty$ an infinite number of times as $\tilde{\beta} h_{b}$ varies, we can conclude that there will be an infinite number of discrete modes in the upper (bubbly) layer. For example, in the limiting case of $H \rightarrow h \rightarrow h_{b}$ (i.e. there is only the bubble layer), (54) gives us $\sin \tilde{\beta} h_{b}=0$, so that $\tilde{\beta}_{n}=n \pi / h_{b}, n=1,2,3 \ldots$ We now notice, that since $N_{2}=0$, then (55) coincides with the dispersion relation (29) now solved for $k^{2}$ as a function of the frequency $\omega$ and the vertical wavenumber $\tilde{\beta}$. Therefore, the eigenfrequencies are given by the dispersion relation found in section 3 , where the vertical wavenumber $\tilde{\beta}$ is quantized to the restricted set of values defined by (54). For example, in the limiting case of $H \rightarrow h \rightarrow h_{b}$, for each value of $\tilde{\beta}$ there are two branches of the dispersion relation, the "internal" wave and the "bubble" wave (see section 3). For the "internal" wave, the frequency lies below a value slightly lower than $N_{1}$; for the "bubble" wave, the frequency is close to $N_{1}$ for very long waves, but typically, is much higher (see section 3 for details). Note that $N_{1}$ is the effective buoyancy frequency in the upper (bubbly) layer, and since we assume here that there is no background stratification, the effective buoyancy frequency is given by

$$
N_{1}^{2} \approx g \alpha_{g 0}\left(\ln n_{0}\right)_{z}
$$

For example, for $\alpha_{g 0}=5 \cdot 10^{-6}, N_{1} \approx 7 \cdot 10^{-3} s^{-1}$. Thus, the presence of a depth-dependent distribution of bubbles alone leads to the possibility of existence of two classes of waves (bubble waves and internal waves) in the upper (bubbly) layer. 


\section{Conclusion}

In this paper we have described a study of the influence of an upper bubbly layer on the properties of the oceanic waveguide. We have considered a simplified configuration, in which the horizontal variability of bubble distributions in a real oceanic environment has been ignored in favour of a study of the consequences of the depth-dependent distribution of bubbles. Further, in this simplified model, the depth-dependence of the actual bubble layer was taken into account only through the effective buoyancy frequency, while the remaining variable coefficients in the equations of motion were replaced by their average values over the depth of the bubble layer. A three-layer model has been used to account for the mismatch between the depth of the bubble layer and the depth of the upper mixed layer. As follows from our analysis, the presence of the vertically-variable distribution of bubbles introduces an effective stratification and essentially changes the value of the buoyancy frequency in the upper part of the ocean, which should be replaced by an effective value. Further, the presence of the upper bubbly layer causes a major change to the dispersion relation, in that a "bubble" mode is introduced along with the internal waves, which typically has a higher frequency than the buoyancy frequency (except possibly for very long waves). Also, we have shown that the presence of a depth-dependent distribution of bubbles alone leads to the possibility of existence of two classes of waves (bubble waves and internal waves) in the upper (bubbly) layer. There is usually a spectral gap between the "bubble" waves and the internal waves in the bubbly layer, with the effective buoyancy frequency being a lower bound for the "bubble" wave, and the upper bound for the internal wave being a bit less than the effective buoyancy frequency. All this points to the essential influence of the bubble distributions in the upper ocean on the properties of the internal wave field and invites further detailed study.

\section{Acknowledgments}

We are thankful to S.L. Gavrilyuk, R.I. Nigmatulin and L.A. Ostrovsky for useful and stimulating discussions. We appreciate support of the Department of Mathematical Sciences of Loughborough Universtiy.

\section{References}

[1] d'Agostino, L. and C.E. Brennen, 1989: Linearized dynamics of spherical bubble clouds. J. Fluid Mech., 199, 155-176.

[2] Akhatov, I.Sh., 1989: The shear instability in stratified viscoelastic and liquid-gas media. J. Appl. Maths Mechs, 53, N4, 630-635.

[3] Brennen, C.E., 1995: Cavitation and bubble dynamics. Oxford University Press, 282 pp.

[4] Brennen, C.E. 1998: Cloud cavitation: observations, calculations and shock waves. Multiphase Sci. and Technology, 10, 303-321. 
[5] Buckingham, M.J., 1997: Sound speed and void fraction profiles in the sea surface bubble layer. Applied Acoustics, 51, 3, 225-250.

[6] Bullock, G.N., Crawford, A.R., Hewson, P.J., Walkden, M.J.A., and P.A.D. Bird, 2001: The influence of air and scale on wave impact pressures. Coastal Engineering, 42, 291-312.

[7] Crawford, G.B. and D.M. Farmer, 1987: On the spatial distribution of ocean bubbles. J. Geophys. Res., 92, 8231-8243.

[8] Dean, G.B. and M.D. Stokes, 1999: Air entraiment processes and bubble size distributions in the surf zone. J. Phys. Oceanogr., 29, 1393-1403.

[9] Druzhinin, O.A., Ostrovsky, L.A., and A. Prosperetti, 1996: Low-frequency acoustic wave generation in a resonant bubble-layer.J. Acoust. Soc. Am., 100, 3570.

[10] Farmer, D.M. and D.D. Lemon, 1984: The influence of bubbles on ambient noise in the ocean at high wind speeds. J. Phys. Oceanogr., 14, 1762-1778.

[11] Farmer, D.M. and S. Vagle, 1989: Waveguide propagation of ambient sound in the ocean-surface bubble layer. J. Acoust. Soc. Am., 86, 1897-1908.

[12] Farmer, D.M., Vagle, S., and A.D. Booth, 1997: A free-flooding acoustical resonator for measurment of bubble size distributions. J. Atmos. and Oceanic Technol., 15, 1132-1146.

[13] Farmer, D.M., Vagle, S., and M. Li, 1999: Wave breaking, turbulence and bubble distributions in the ocean surface layer. Proceedings, The wind-driven air-sea interface, M.L. Banner, Ed., The University of New South Wales, Sydney, Australia, 187-192.

[14] Garrett, C., Li, M., and D.M. Farmer, 2000: The connection between bubble size and energy dissipation rates in the upper ocean. J. Phys. Oceanogr., 30, 2163-2171.

[15] Gill, A.E., 1982: Atmosphere - Ocean Dynamics, Academic Press, 662 pp.

[16] Grimshaw, R.H.J. and K.R. Khusnutdinova, 2004: The effect of bubbles on internal waves. J. Phys. Oceanogr. 34, 477-489.

[17] Iordansky, S.V., 1960: Equations of motion of liquid containing gas bubbles. Zh. Prikl. Mekh. Tekh. Fiz., 3, 102-110 (in Russian).

[18] Johnson, B.D. and R.C. Cooke, 1979: Bubble populations and spectra in coastal waters: A photographic approach. J. Geophys. Res., 84, 3761-3766.

[19] Karpov, S., Prosperetti, A., and L. Ostrovsky, 2003: Nonlinear wave interactions in bubble layers, J. Acoust. Soc. Am., 113, 3, 1304-1316.

[20] Kogarko, B.S., 1961: On the model of cavitating liquid. Dokl. AN SSSR, 137, 13311333 (in Russian).

[21] Kolovaev, P.A., 1976: Investigation of the concentration and statistical size distribution of wind-produced bubbles in the near surface ocean layer. Oceanology, 15, 659-661.

[22] Kumar, S. and C.E. Brennen, 1993: Some nonlinear interactive effects in bubbly clouds. J.Fluid Mech., 253, 565-591. 
[23] Kutateladze, S.S. and V.E. Nakoryakov, 1984: Heat and mass-transfer and waves in gas-liquid systems. Nauka, 301 pp (in Russian).

[24] Medwin, H., 1974: Acoustic fluctuations due to microbubbles in the near-surface ocean. J. Acoust. Soc. Am., 56, 1100-1104.

[25] Medwin, H., 1977: In situ acoustic measurements of microbubbles at sea. J. Geophys. Res., 82, 971-976.

[26] Melville, W.K., 1996: The role of surface-wave breaking in air-sea interaction. Annu. Rev. Fluid Mech., 28, 279-321.

[27] Minnaert, M., 1933: On musical air bubbles and the sounds of running water. Philos. Mag., 16, 235-248.

[28] Nakoryakov, V.E., Pokusaev, V.G., and I.R. Shreiber, 1983: Propagation of waves in gas- and vapour-liquid media. Institute of Thermorhysics, Novosibirsk, $280 \mathrm{pp}$ (in Russian).

[29] Naugolnykh, K. and L. Ostrovsky, 1998: Nonlinear wave processes in acoustics, Cambridge University Press, 298 pp.

[30] Nigmatulin, R.I., 1991: Dynamics of multiphase media, Vol.1 \& 2. Hemisphere, 507 $\& 371 \mathrm{pp}$.

[31] Ostrovsky, L.A., Sutin, A.M., Soustova, I.A., Matveyev, A.I., and A.I. Potapov, 1998: Nonlinear, low-frequency sound generation in a bubble layer: Theory and laboratory experiment. J. Acoust. Soc. Am., 104, 722-726.

[32] Plesset, M.S. and A. Prosperetti, 1977: Bubble dynamics and cavitation. Ann. Rev. Fluid Mech., 9, 145-185.

[33] Prosperetti, A., 1994: Bubble dynamics: some things we did not know 10 years ago. Bubble Dynamics and Interface Phenomena, J. Blake, J. Boulton-Stone \& N. Tomas, Ed., Kluwer, 3-16.

[34] Rayleigh, Lord, 1917: The pressure developed in a liquid on the collapse of a spherical cavity. Phyl. Mag., 34, 94.

[35] Reisman, G.E., Wang, Y.C., and C.E. Brennen, 1998: Observation of shock waves in cloud cavitation. J. Fluid Mech, 355, 255-283.

[36] Scott, J.C., 1975: The role of salt in whitecap persistence. Deep-Sea Research, 22, 653-657.

[37] Terrill, E.J. and W.K. Melville, 1999: Field measurements of bubble size distributions in the upper mixed layer. Proceedings, The wind-driven air-sea interface, M.L. Banner, Ed., The University of New South Wales, Sydney, Australia, 247-255.

[38] Thorpe, S.A., 1982: On the clouds of bubbles formed by breaking wind-waves in deep water, and their role in air-sea gas transfer. Phil. Trans. R. Soc. Lond. A, 304, 155-210.

[39] Thorpe, S.A., 1984a: On the determination of $K_{v}$ in the near-surface ocean from acoustic measurments of bubbles. J. Phys. Oceanogr., 14, 855-863. 
[40] Thorpe, S.A., 1984b: A model of the turbulent diffusion of bubbles below the sea surface. J. Phys. Oceanogr., 14, 841-853.

[41] Thorpe., S.A., 1995: Dynamical processes of transfer at the sea surface. Prog. Oceanogr., 35, 315-352.

[42] Thorpe, S.A. and A.J. Hall, 1983: The characteristics of breaking waves, bubble clouds, and near-surface currents observed using side- scan sonar. Continental Shelf Research, 1, 4, 353-384.

[43] Thorpe, S.A., Belloul, M.B., and A.J. Hall, 1987: Internal waves and whitecaps. Nature, 330, 740-742.

[44] Urick, R.J., 1983: Principles of underwater sound, McGraw-Hill, 384 pp.

[45] van Wijngaarden, L., 1968: On the equations of motion for mixtures of liquid and gas bubbles. J. Fluid Mech., 33, 465-474.

[46] Woolf, D.K. and S.A. Thorpe, 1991: Bubbles and the air-sea exchange of gases in near saturation conditions. J. Mar. Res., 49, 435-466.

[47] Zedel, L. and D. Farmer, 1991: Organized structures in subsurface bubble clouds: Langmuir circulation in the open ocean, J. of Geophys. Res., 96, C5, 8889-8900. 